Research Paper

\title{
Eicosapentaenoic acid reduces indoleamine 2,3-dioxygenase 1 expression in tumor cells
}

\author{
Chih-Chiang Wang ${ }^{1}$, Chih-Jen Yang 2,3,4, Li-Hsien Wu ${ }^{5}$, Han-Chen Lin ${ }^{6}$, Zhi-Hong Wen7, Che-Hsin Lee ${ }^{8,9} \bowtie$ \\ 1. Division of Nephrology, Department of Internal Medicine, Kaohsiung Armed Forces General Hospital, Kaohsiung, Taiwan \\ 2. Department of Internal Medicine, Kaohsiung Municipal Ta-Tung Hospital, Kaohsiung Medical University, Kaohsiung, Taiwan \\ 3. Division of Pulmonary and Critical Care Medicine, Department of Internal Medicine, Kaohsiung Medical University Hospital, Kaohsiung Medical \\ University, Kaohsiung, Taiwan \\ 4. Faculty of Medicine, Department of Respiratory Therapy, College of Medicine, Kaohsiung Medical University, Taiwan \\ 5. Department of Public Health, China Medical University, Taichung, Taiwan \\ 6. Department of Anatomy, School of Medicine, College of Medicine, Kaohsiung Medical University, Kaohsiung, Taiwan \\ 7. Department of Marine Biotechnology and Resources, National Sun Yat-sen University, Kaohsiung, Taiwan \\ 8. Department of Biological Sciences, National Sun Yat-sen University, Kaohsiung, Taiwan \\ 9. Department of Medical Research, China Medical University Hospital, China Medical University, Taichung, Taiwan. \\ $\square$ Corresponding author: Dr. Che-Hsin Lee, Department of Biological Sciences, National Sun Yat-sen University, Kaohsiung, Taiwan, 70 Lienhai Rd. Kaohsiung \\ 80424, Taiwan. E-mail: chlee@mail.nsysu.edu.tw
}

(C) Ivyspring International Publisher. This is an open access article distributed under the terms of the Creative Commons Attribution (CC BY-NC) license (https://creativecommons.org/licenses/by-nc/4.0/). See http://ivyspring.com/terms for full terms and conditions.

Received: 2018.05.17; Accepted: 2018.07.26; Published: 2018.08.06

\begin{abstract}
Marine plants and animals have omega-3 fatty acids including eicosapentaenoic acid (EPA) and docosahexaenoic acid (DHA). EPA is required for biological processes, but humans are unable to synthesize them and must be obtained from dietary sources. EPA has been used as an antitumor agent but the molecular mechanisms for the regulation of tumor microenvironment immunity by EPA are still unknown. The indoleamine 2,3-dioxygenase 1 (IDO) catalyzes conversion of tryptophan to kynurenine to induce immune evasion in tumor microenvironment. In this study, EPA inhibited the expression of IDO via downregulation of protein kinase B (Akt)/mammalian targets of rapamycin (mTOR) signaling pathway in tumor cells. Meanwhile, a significant decrease in kynurenine levels and increase in T cell survival were observed after tumor cells treated with EPA. The results demonstrated that EPA can activate host antitumor immunity by inhibiting tumor IDO expression. Therefore, our finding suggests that EPA can be enormous potential for cancer immunotherapy.
\end{abstract}

Key words: Eicosapentaenoic acid; tumor microenvironment; indoleamine 2,3-dioxygenase; immune evasion; immunotherapy

\section{Introduction}

Indoleamine 2,3-dioxygenase (IDO) is involved in the tryptophan-kynurenine pathway that catalyzes conversion of tryptophan to kynurenine. IDO induction results in tryptophan depletion and reduced T cell proliferation [1]. Tryptophan depletion can induce uncharged tryptophan-tRNA, regulator T cell activation, and prevent effector $\mathrm{T}$ cell proliferation [2]. IDO can promote tumor immune escape. IDO expression was found in tumor cells including melanoma and breast cancer [3].

Marine-derived $\omega-3$ polyunsaturated fatty acids (PUFAs) are involved in cell growth and membrane lipid composition [4]. Marine-based fish and fish oil contain a high proportion of $\omega-3$ PUFAs, which are known to have variety of health benefits against cardiovascular diseases, tumor, depression, aging, and arthritis. Dietary intake of marine original $\omega-3$ PUFAs has been found good effects for cognitive abilities [5]. The absorption of $\omega-3$ PUFAs is influenced by food intake and reduced bioavailability. The bioavailability of $\omega-3$ PUFAs is important for body health, supplying not only energy, but also taking part in host immunity. The bioavailability of $\omega-3$ PUFAs is associated with many factors, including genetic background, living habit, and gut microbiota [6]. Previous studies also have shown that fish 
consumption and dietary intake of $\omega$-3 PUFAs are inversely associated with the risk of colorectal cancer [4]. Meanwhile, some studies have demonstrated that chronic inflammation can be reduced after $\omega-3$ PUFAs intake [5]. Marine $\omega-3$ PUFAs, including eicosapentaenoic acids (EPA) and docosahexaenoic acids (DHA), have been shown to reduce inflammation and growth of tumors. EPA can synthesize DHA in the body. Recently, EPA and DHA can restore the inflammation-induced increase in kynurenine pathway. EPA has the better activity than DHA to reduce the damage induced by inflammation in neurons [7]. EPA is more effective than DHA in antidepressant clinical trials [8]. However, whether marine original EPA can increase the activity of host immune cells against tumor cells is unclear. EPA can prevent tumor growth and has antitumor activity. Increasing evidence suggest that EPA could limit the response to host unwanted immune activities including cardiac disease, bronchial disorders, and intestinal inflammation $[9,10]$. EPA is linked between host immunity and tumor. The detailed molecular mechanism is in urgent need of strengthening. In this study, we want to examine whether EPA can overcome the immune tolerance of tumor microenvironment by downregulating IDO.

\section{Materials and Methods}

\section{Cell lines, reagents and plasmid}

Murine B16F10 and 4T1 cells are maintained in Dulbecco's Modified Eagle's medium (DMEM) containing $10 \%$ fetal bovine serum (FBS), 1\% Penicillin-Streptomycin (100 units/mL penicillin and $100 \mathrm{\mu g} / \mathrm{mL}$ streptomycin), and at $37^{\circ} \mathrm{C}$ in $5 \% \mathrm{CO}_{2}$ [11]. Jurkat $\mathrm{T}$ cell line (human $\mathrm{T}$ lymphocyte; (ATCC TIB-152 ${ }^{\mathrm{TM}}$ )) cultured in HyClone RPMI 1640 medium containing 10\% FBS. EPA, and L-3,4-dihydroxyphenylalanine (L-DOPA) were purchased from Sigma-Aldrich (Sigma Aldrich, St. Louis, MO, USA). The constitutively phosphorylation AKT plasmid (myr-AKT) mentioned previously [11].

\section{Western blotting}

Cells were collected and lysed in NP-40 buffer. The bicinchoninic acid (BCA) protein assay (Pierce Biotechnology, Rockford, IL, USA) was used to measure the protein content. Proteins were separated by using SDS-PAGE (8\%) and transferred to nitrocellulose membranes [12]. The primary antibodies IDO (Thermo Scientific, Rockford, IL, USA), mTOR (Cell Signaling, Danvers, MA, USA), phosphorylation-mTOR (Cell Signaling), protein kinase B (AKT) (Santa Cruz Biotechnology, Inc. Santa Cruz, CA, USA), phosphorylation-AKT (Santa Cruz
Biotechnology, Inc.), p70S6K (Cell Signaling), phosphorylation-p70S6K (Cell Signaling), and $\beta$-actin (Sigma Aldrich)) were used to detect the protein expression. Anti-murine or -rabbit secondary antibodies were used to recognize primary antibodies. The enhanced chemiluminescence system was used to visualize the protein expressions and signals were quantified with ImageJ software [13].

\section{$\mathbf{T}$ cell viability assay}

Tumor cells were treated with various concentrations EPA $(50-200 \mu \mathrm{M})$ for $24 \mathrm{~h}$. The conditioned medium from EPA-treated B16F10 or 4T1 cells were collected and cultured with Jurkat $\mathrm{T}$ cells ( 2 $\times 10^{5}$ ) for $72 \mathrm{~h}$. The number of cell was measured by staining trypan blue or WST-8 [13].

\section{Kynurenine assay}

B16F10 and 4T1 cells were treated with EPA for $24 \mathrm{~h}$. The supernatants were collected and mixed with TCA (Sigma-Aldrich). The samples were added the Erchlich' s reagent (Sigma-Aldrich). The absorption was measured at $490 \mathrm{~nm}$. The data were performed as a percentage of control.

\section{Transfection assay}

Lipofectamine 2000 (Thermo Scientific) was used to transfect myr-AKT plasmids to B16F10 and 4T1 cells. After transfecting and EPA treating, cells were harvested and determined the protein expression, $\mathrm{T}$ cell number and kynurenine content.

\section{Statistical analysis}

The ANOVA was used to determine differences between groups. Any $\mathrm{P}$ value less than 0.05 is regarded statistically significant.

\section{Results}

\section{EPA inhibited the accumulation of kynurenine and promoted the viability of $\mathbf{T}$ cells in vitro}

The high level of kynurenine resulted in $\mathrm{T}$ cell proliferation [14]. In this study, we used mouse B16F10 melanoma cells and mouse breast tumor 4T1 cells to evaluate the downregulation kynurenine activity of EPA. After tumor cells were treated with EPA and the tumor cell viability is shown in Fig. 1 A, B E. By comparison with trypan blue exclusion assay and WST-8 assay, there were no significant differences between the two cell viability methods (Fig. $1 \mathrm{C}$ and D). The trypan blue exclusion assay was used in the subsequent cell viability assay. The dose $(50 \mu \mathrm{M}-200 \mu \mathrm{M})$ without inducing cytotoxicity were chosen to determine the influence of EPA on kynurenine downregulation (Fig. 2). After EPA treatment, the production of kynurenine was 
A B16F10

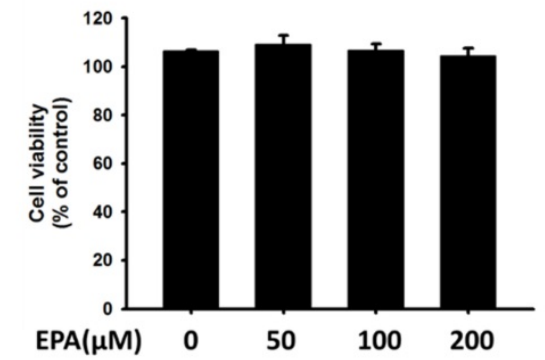

B $\quad 4 T 1$

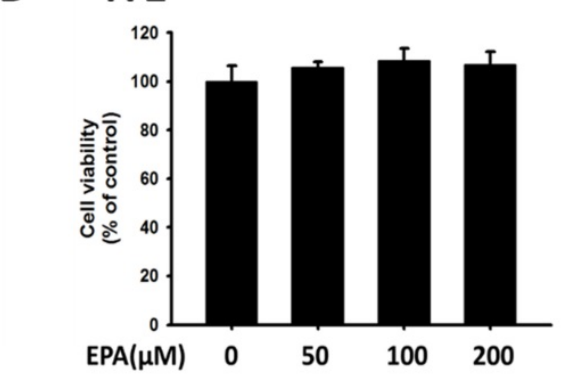

C B16F10

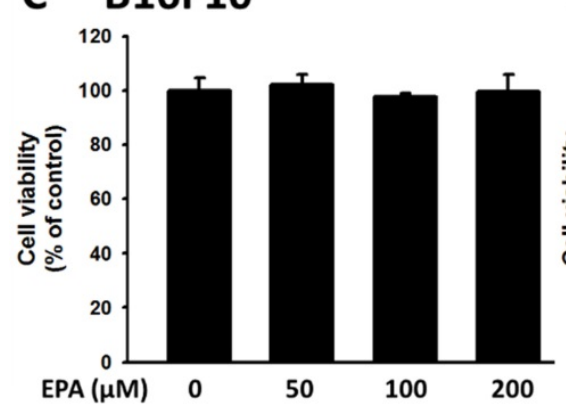

D $\quad 4 T 1$

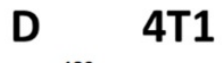

Figure 1. Effects of EPA on tumor cell viability. B16F10 (A) and 4T1 (B) cells were treated with EPA (50-200 $\mu M)$ for $24 \mathrm{~h}$. The number of cell was measured by the trypan blue exclusion assay. B16F10 (C), 4T1 (D) and Jurkat T (E) cells were treated with EPA (50-200 $\mu \mathrm{M})$ for $24 \mathrm{~h}$. The number of cell was measured by WST-8 assay.

significantly reduced in B16F10 and 4T1 cells (Fig. 2 A and B). The contents of kynurenine in two tumor cells were downregulated after treatment with the highest dose of EPA $(200 \mu \mathrm{M})$. The decreasing production of kynurenine by treating EPA is a general phenomenon. Furthermore, we measured whether EPA-mediated decrease of kynurenine has an effect on the viability of $\mathrm{T}$ cells. The $\mathrm{T}$ cells treated with the conditioned medium of EPA-treated tumor cells and cell viability was observed (Fig. $2 \mathrm{C}$ and D). The highest numbers of $\mathrm{T}$ cells were observed in culturing with condition medium treated with EPA $(200 \mu \mathrm{M})$ group (Fig. $2 \mathrm{C}$ and D). For the interpretation of the results it is essential to examine the effect of medium from EPA-untreated cancer cells on $\mathrm{T}$ cell viability in comparison to the control medium. Fig 3 shows the conditioned medium from EPA-treated tumor cells enhanced the viability of T cell. When taken together, EPA did not inhibit tumor growth but enhanced the viability of $\mathrm{T}$ cells by reducing the accumulation of kynurenine.

\section{EPA dose-dependently inhibited IDO expression}

EPA could decrease the degradation of tryptophan to kynurenine and reverse tryptophan-depleted $\mathrm{T}$ cell death. IDO is a rate-limiting enzyme in the tryptophan-kynurenine pathway [15]. Fig. $4 \mathrm{~A}$ and B show EPA reduced the expression of IDO in a dose-dependent manner in B16F10 and 4T1 cells. Previously, the protein kinase B (Akt)/mammalian targets of rapamycin (mTOR)/p70 ribosomal s6 kinase (p-p70s6K) regulated the expression of IDO [14]. Indeed, the expressions of phosphorylation AKT, phosphorylation mTOR, and phosphorylation p70s6K were reduced after EPA treatment in a dose dependent-manner (Fig. $4 \mathrm{~A}$ and B). We observed the correlation between IDO and the AKT/mTOR signaling pathway. These results suggested that EPA might inhibit IDO through reducing the activity of $\mathrm{AKT} / \mathrm{mTOR}$ signaling pathway. The conditioned medium from EPA-treated tumor cells not only increased the $\mathrm{T}$ cell viability but also activated the AKT/mTOR signal pathway in $\mathrm{T}$ cells (Fig. 5). In contrast to EPA-treated tumor cells, the upregulation of $\mathrm{AKT} / \mathrm{mTOR}$ signal pathway expression was observed in the conditioned medium-treated T cells.

\section{EPA inhibited IDO expression through the suppression of the AKT/mTOR signaling pathway}

EPA reduced the expression of IDO and the phosphorylation AKT in our system. Furthermore, the constitutively active AKT plasmids were used to distinguish the correlation between IDO and the AKT/mTOR signaling pathway. Transfecting constitutively active AKT plasmids reversed the AKT/mTOR/p70S6K signaling pathway (Fig. 6 A and $\mathrm{B})$. The phenomenon was reversed after being treated with EPA and constitutively AKT in B16F10 and $4 \mathrm{~T} 1$ cells, indicating that the EPA-regulated decrease of IDO via AKT/mTOR/p70S6K signaling pathway. The expression of IDO significantly 
A B16F10

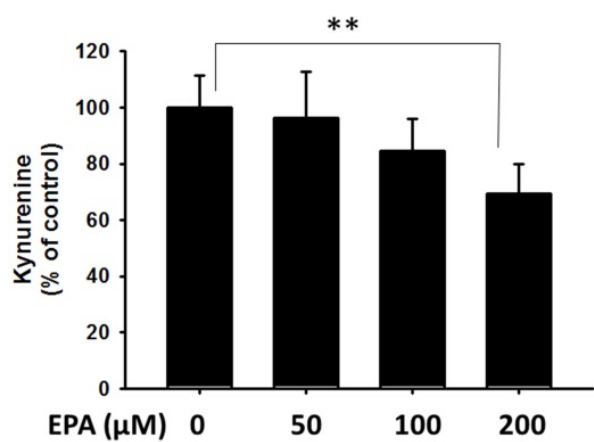

C B16F10

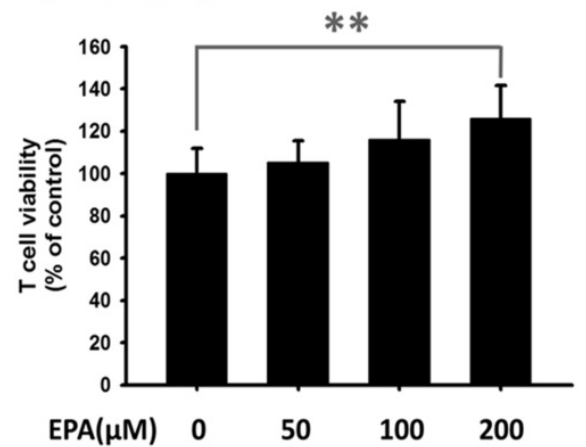

B

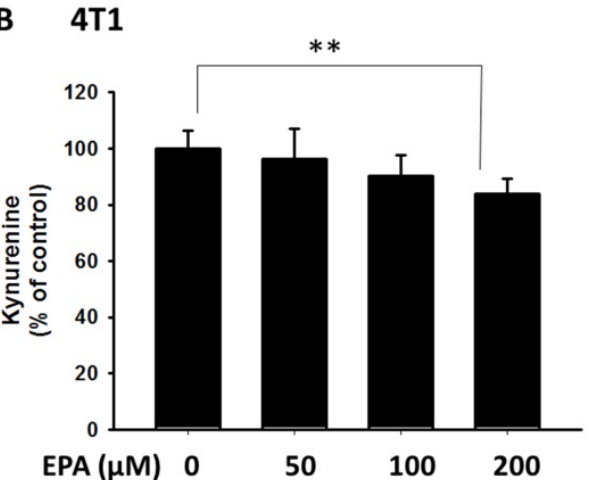

D $\quad 4 T 1$

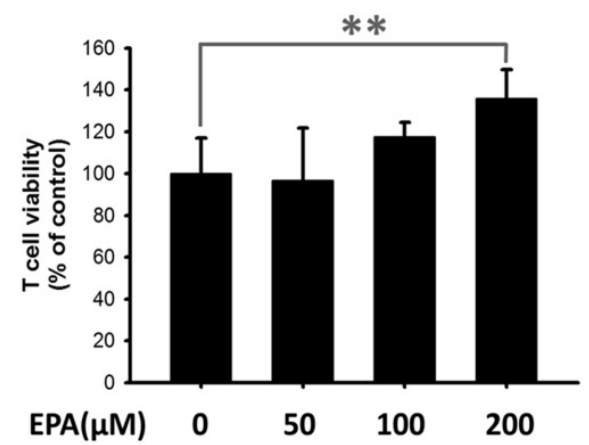

Figure 2. The accumulation of kynurenine and T cell proliferation were measured after EPA treatment. The kynurenine assay was used for the kynurenine production in B16F10 (A) and 4T1 (B) cells. The conditioned medium of B16F10 (C) and 4T1 (D) after treated with indicated concentrations of EPA for $24 \mathrm{~h}$ mixed with an equal amount of original medium. $T$ cells were cultured in media conditioned from tumor cells for $72 \mathrm{~h}$. The cell number were measured by staining with trypan blue. $(\mathrm{n}=6$, data are mean \pm SD. $* * *$ $0.01)$.

reversed in AKT-transfecting cells after EPA treatment (Fig. 6). The results point out that downregulation of phosphorylation AKT is essential for EPA-mediated IDO expression in tumor cells. Similar results were observed and showed that EPA inhibited the phosphorylation AKT in MDA-MB-231 breast tumor cells [16].

\section{EPA reduced kynurenine content and enhanced $T$ cell viability through AKT pathway}

EPA decreased IDO expression in B16F10 and $4 \mathrm{~T} 1$ cells through reducing the phosphorylation AKT. The AKT/mTOR signaling pathway can be reversed by transfecting a plasmid bearing a constitutively active form of AKT [17]. Transfection of a plasmid encoding constitutively active AKT increased the production of kynurenine and reduced the $\mathrm{T}$ cell viability (Fig. 7). EPA decreased the content of kynurenine in B16F10 and 4T1 cells (Fig. 7). The cells treated with EPA and constitutively active AKT plasmids restored the expression of IDO compared with those cells treated with EPA only. Our results indicated that inhibition of phosphorylation-AKT is necessary for the reduction of kynurenine production and the enhancement of T cell viability in B16F10 and $4 \mathrm{~T} 1$ cells treated with EPA.

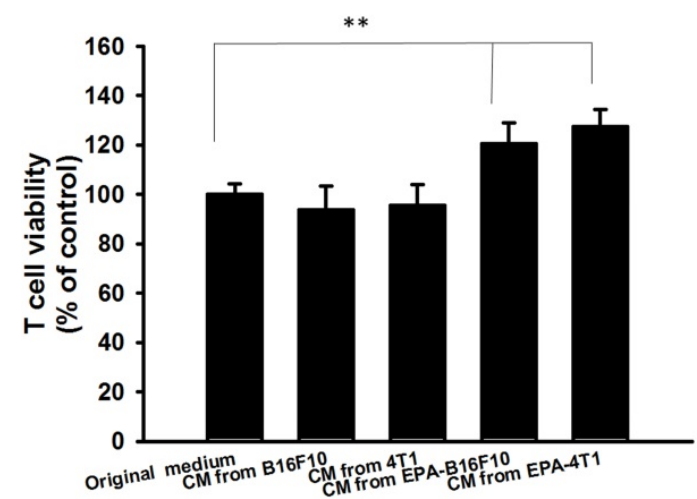

Figure 3. Effects of EPA on T cell viability. The conditioned medium of $B 16 \mathrm{~F} 10$ and $4 \mathrm{~T} 1$ after treated with EPA $(0$ or $200 \mu \mathrm{M})$ for $24 \mathrm{~h}$ mixed with an equal amount of original medium or not. The media were cultured with $T$ cell for $72 \mathrm{~h}$. The cell number were measured by staining with trypan blue. $(n=6$, data are mean $\pm S D$. ** $P$ $<0.01)$. CM: conditioned medium.

\section{Discussion}

Tumor microenvironments can affect behavior and clinical outcome of cancer patients. An efficient antitumor agent is impeded by tumor immune tolerance. To put up with this immune evasion, host immune cells recognize and kill tumor cells by an effective agent. Tumor expressed IDO has been observed in different tumors including melanoma, breast cancer, and colon cancer [3]. This study aims to identify that EPA as an immunotherapy agent 
discouraged tumor IDO further diffusion. The AKT/mTOR pathway was involved in multiple tumor growth signaling pathways [18]. Indeed, EPA treatment suppressed the IDO expression in tumor cells. Moreover, growing evidences indicate that AKT/mTOR pathways are involved in immune regulation [19]. This study learned about the

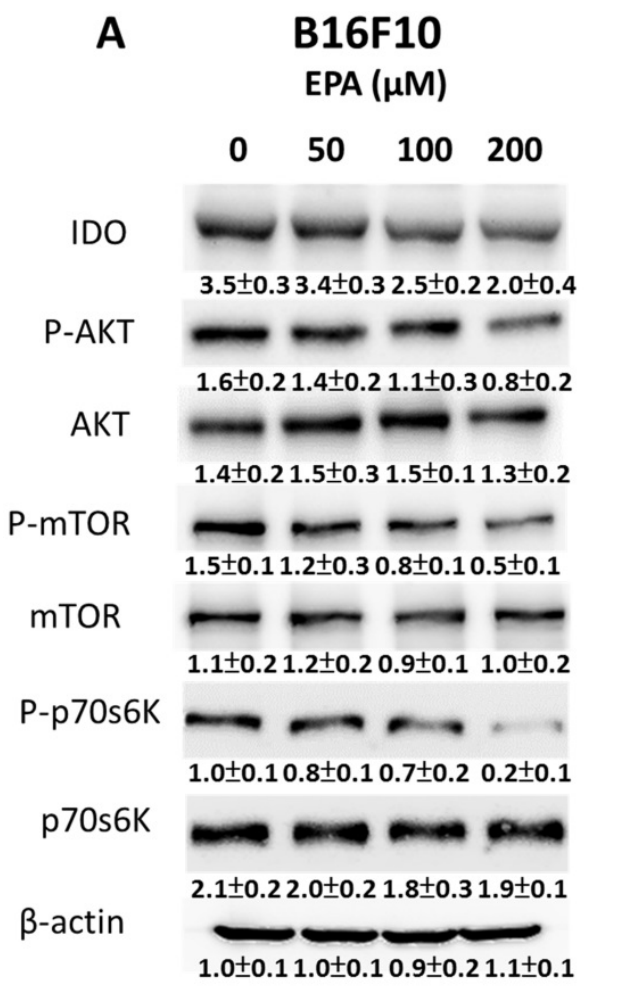

relationship between AKT/mTOR signaling pathway and IDO was confirmed by using constitutively-AKT transfecting experiments. The constitutively-AKT activity reversed the EPA-reduced IDO expression (Fig. 6), which indicated that EPA reduced IDO expression via $\mathrm{AKT} / \mathrm{mTOR}$ pathway.

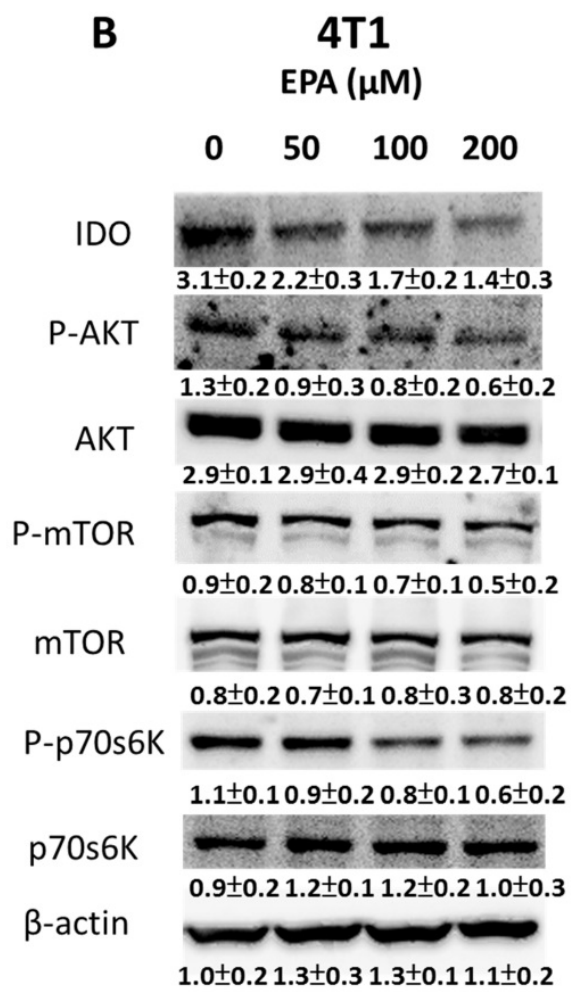

Figure 4. EPA mediated IDO via AKT/mTOR signaling pathway. The tumor cells were treated with of EPA for $24 \mathrm{~h}$. The BI6F10 (A) and 4TI (B) cells were collected and measured for IDO, and AKT/mTOR/p70S6K by Western blotting. The Immunoblotting assay was repeated three times with similar results. Inset values show the protein expression normalized to $\beta$-actin. $(n=3$, data are mean $\pm S D)$.

The supernatant from B16F10

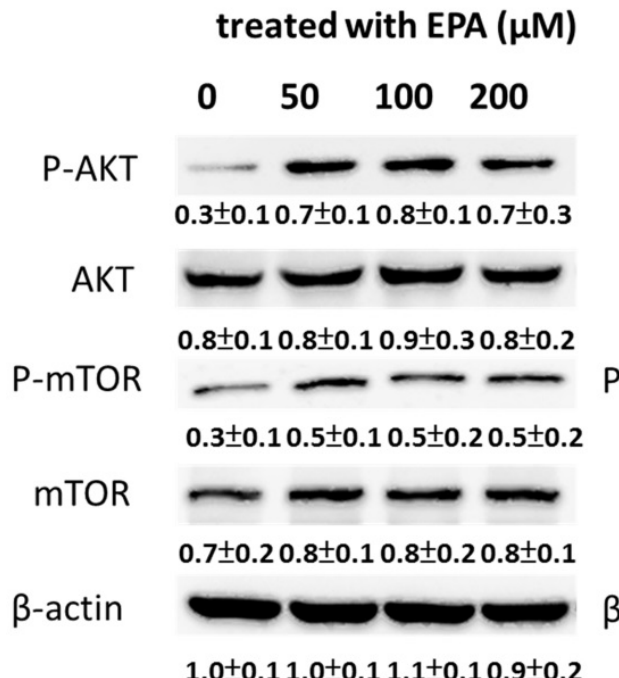

The supernatant from 4T1

\section{treated with EPA $(\mu \mathrm{M})$}

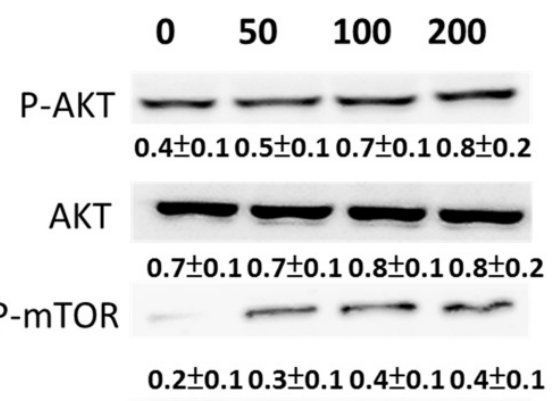

mTOR

$0.8 \pm 0.10 .8 \pm 0.10 .8 \pm 0.20 .8 \pm 0.1$

$\beta$-actin

Figure 5. EPA induced AKT/mTOR signaling pathway in T cells. The conditioned medium of B16F10 and $4 \mathrm{~T} 1$ after treated with EPA ( 0 or $200 \mu \mathrm{M})$ for $24 \mathrm{~h}$ mixed with an equal amount of original medium or not. The media were cultured with T cell for $72 \mathrm{~h}$. The T cells were collected and measured for AKT and mTOR by Western blotting. The Immunoblotting assay was repeated three times with similar results. Inset values show the protein expression normalized to $\beta$-actin. $(\mathrm{n}=3$, data are mean \pm SD). 
A

B16F10

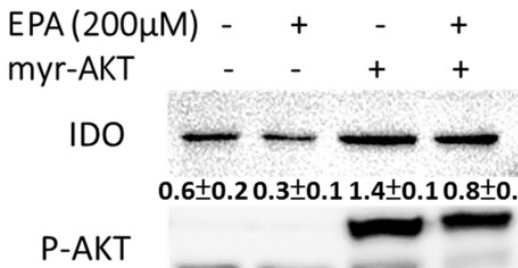

$0.3 \pm 0.10 .2 \pm 0.13 .1 \pm 0.52 .5 \pm 0.3$

AKT

P-mTOR

mTOR

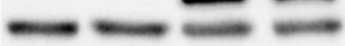

$1.4 \pm 0.11 .3 \pm 0.12 .9 \pm 0.22 .7 \pm 0.1$

$0.7 \pm 0.20 .3 \pm 0.10 .9 \pm 0.20 .4 \pm 0.1$

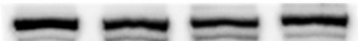

$1.3 \pm 0.10 .7 \pm 0.11 .9 \pm 0.41 .2 \pm 0.2$

P-p70s6K

$1.3 \pm 0.10 .7 \pm 0.11 .9 \pm 0.41 .2 \pm 0.1$

p70s6K

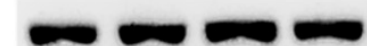

$1.4 \pm 0.21 .5 \pm 0.21 .5 \pm 0.11 .4 \pm 0.3$

$\beta$-actin
$1.0 \pm 0.11 .1 \pm 0.21 .1 \pm 0.11 .2 \pm 0.1$
B

\section{T1}

$\begin{array}{lllll}\text { EPA }(200 \mu \mathrm{M}) & - & + & - & + \\ \operatorname{myr}-\mathrm{AKT} & - & - & + & +\end{array}$

IDO

P-AKT

$2.0 \pm 0.41 .3 \pm 0.42 .2 \pm 0.1 \quad 1.5 \pm 0.4$

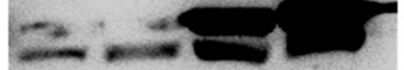

$0.7 \pm 0.3 \quad 0.6 \pm 0.34 .2 \pm 0.7 \quad 5.1 \pm 0.5$

AKT

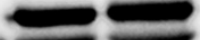

$0.9 \pm 0.10 .8 \pm 0.22 .2 \pm 0.3 \quad 1.9 \pm 0.4$

P-mTOR

$\begin{array}{lll}0.7 \pm 0.2 & 0.5 \pm 0.10 .9 \pm 0.2 & 0.6 \pm 0.2\end{array}$

mTOR

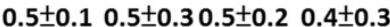

P-p70s6K

$\begin{array}{lll}0.6 \pm 0.3 & 0.2 \pm 0.31 .2 \pm 0.2 & 1.1 \pm 0.2\end{array}$

p70s6K

$0.9+ \pm 0.20 .8 \pm 0.31 .0 \pm 0.1 \quad 1.0 \pm 0.1$

$\beta$-actin

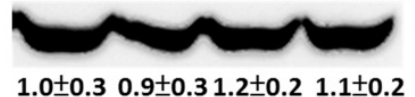

Figure 6. Constitutively active-AKT reduced EPA-induced decrease of IDO. The B16F10 and 4T1 cells were transfected with constitutively active AKT plasmids for $16 \mathrm{~h}$ prior to treatment with EPA for $24 \mathrm{~h}$. The expression of IDO, phosphorylation AKT, AKT, phosphorylation mTOR, mTOR, phosphorylation p70s6K, and p70s6K protein in B16F10

$(A)$ and $4 \mathrm{~T} 1$ cells $(B)$ was determined. The Immunoblotting assay was repeated three times with similar results. Inset values show the protein expression normalized to $\beta$-actin. $(\mathrm{n}=3$, data are mean $\pm \mathrm{SD})$.

A

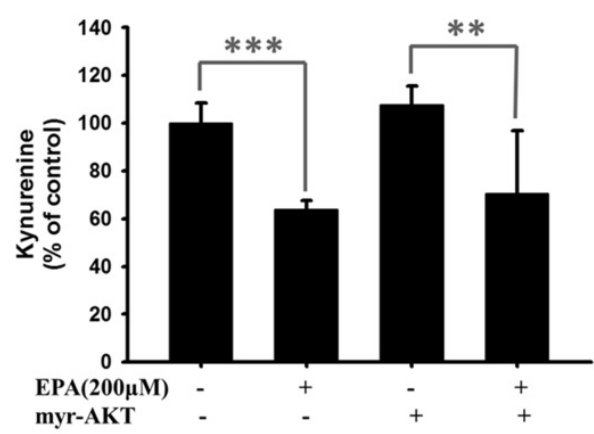

C B16F10

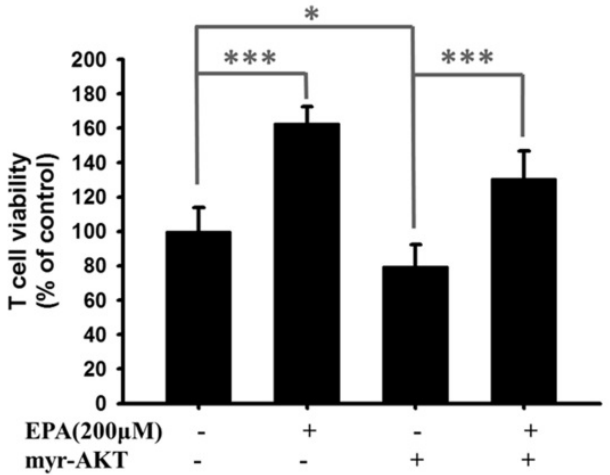

B

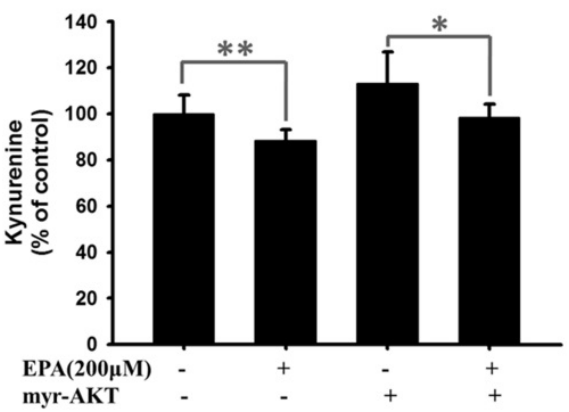

D $\quad 4 \mathrm{~T} 1$

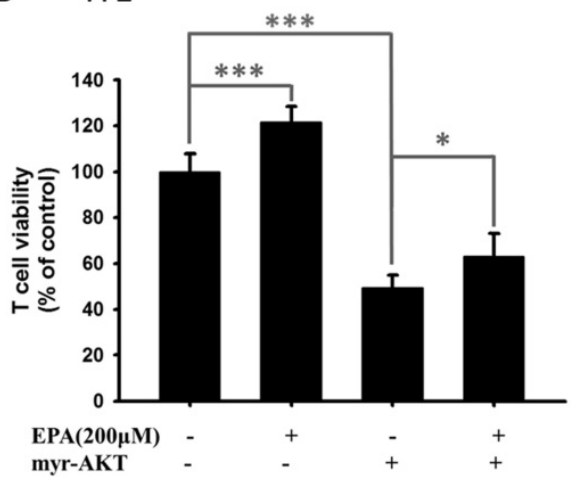

Figure 7. Constitutively phosphorylation-AKT reversed the effect of EPA on the accumulation kynurenine and the viability of T cells. The EPA treated with B16F10 (A, C) and $4 T 1$ (B, D) cells that transfected with constitutively active AKT plasmid. The kynurenine assay was used for the kynurenine production in $B 16 \mathrm{~F} 10$ (A) and 4T1 (B) cells. The conditioned media from cell with various treatments were cultured with $T$ cell for $72 \mathrm{~h}$. The $\mathrm{T}$ cells were collected and measured by staining with trypan blue. ( $\mathrm{n}=6$, data are mean \pm SD. $* P<0.05$; ** $P<0.01$; *** $P<0.001$ ). 
The activation of AKT/mTOR signaling pathway is also involved in cell survival and protects cell against apoptosis and autophagy [20]. The inhibition of Akt/mTOR damages the balance between cell survival and cell death, and enhances tumor cell death [21]. In our system, EPA did not influence the survival of tumor cell (B16F10 and 4T1) and $\mathrm{T}$ cell (Fig. $1 \mathrm{E}$ ), but inhibited the activation of Akt/mTOR signal pathway in tumor cells. Herein, EPA decreased the expression of IDO via Akt/mTOR signal pathway. The conditioned medium from tumor cell treated with EPA increased the survival of T cells through reducing kynurenine, activating Akt/mTOR signal pathway in T cells (Fig. 5). The Akt/mTOR signal pathway is involved in the metabolism of tumor microenvironment.

EPA can directly induce tumor cell death by different pathway in other studies. EPA or in combination with chemotherapy have the ability to retard tumor growth by inducing apoptosis. EPA triggered the intrinsic and extrinsic apoptotic pathways [4]. Meanwhile, some studies have demonstrated that EPA changed the lipid raft and influenced the oncoprotein [22]. EPA caused oxidative stress in tumor cells and disrupted the mitochondrial membrane potential [23].

The activation of Akt/mTOR signaling was observed in the $\mathrm{T}$ cell cocultured with condition-medium derived from EPA-treated tumor cells. The Akt/mTOR pathways is involved the protein synthesis. Jaudszus et al., pointed out that the reduction of intracellular interleukin-2, tumor necrosis factor $-\alpha$, and interleukin- 4 was observed in human T cell after EPA direct treatment [24]. EPA has a great benefit to immunomodulation. Herein, EPA indirectly decreased the proliferation of $\mathrm{T}$ cell. When EPA directly treated with $\mathrm{T}$ cell, the proliferation of $\mathrm{T}$ cell was not influenced (Fig. 1E). The result is consistent with previous report [25]. Some studies also demonstrated EPA decreased the function of antigen-presenting cells (dendritic cells and macrophages) by reducing class II major histocompatibility complex, costimulatory molecules, and IDO expression and depressed the activity of NK cells $[25,26]$.

The immunotherapy is attracting worldwide attention, because some immune checkpoint inhibitors, including anti-program cell death receptor antibody and anti-the cytotoxic T-lymphocyteassociated antigen 4 antibody, has dramatically antitumor activity [27]. IDO inhibitors also had antitumor activity by activating $\mathrm{T}$ cell responses [28]. Some studies suggested that chemotherapy combined with IDO inhibitors, such as 1-methyl tryptophan, seem be encouraged [29]. EPA exerted antitumor activity by inducing caspase-8 signaling pathway [30]. Although EPA did not directly induced tumor cell death in our system, we provided new EPA-targeted molecules: IDO. The concept of using EPA as tumor immune checkpoint inhibitors is attractive, because EPA has been safely used in humans several decades and available at a low cost. These results illustrated the importance of EPA in regulating tumor immune tolerance. The animal tumor models treated with EPA combined with other conventional chemotherapies are worth studying. Meanwhile, our studies pointed out the potential mechanism that EPA reduced tumor IDO expression and enhanced $T$ cell viability. Our findings deserve further investigation to ascertain their potential benefit for EPA in developing immune checkpoints inhibitor.

\section{Acknowledgements}

This work was supported by Kaohsiung Armed Forces General Hospital, Taiwan (107-40) and NSYSU-KMU joint research project, (\#NSYSUKMU 107-P004).

\section{Author Contributions}

C.C.W., C.J.Y. and C.H.L conceived, designed and revised the experiments; C.C.W, C.J.Y, L.H.W, and H.C.L. performed the experiments; C.J.Y. and C.H.L analyzed the data; Z. H.W. and C.H.L contributed reagents/materials/analysis tools; C.J.Y. and C.H.L wrote the paper.

\section{Competing Interests}

The authors have declared that no competing interest exists.

\section{References}

1. Fallarino F, Grohmann U, Puccetti P. Indoleamine 2,3-dioxygenase: from catalyst to signaling function. Eur J Immunol. 2012; 42: 1932-1937.

2. Brochez L, Chevolet I, Kruse V. The rationale of indoleamine 2,3-dioxygenase inhibition for cancer therapy. Eur J Cancer. 2017; 76: 167-182.

3. Lin HC, Yang CJ, Kuan YD, Wang WK, Chang WW, Lee CH. The inhibition of indoleamine 2, 3-dioxygenase 1 by connexin 43. Int J Med Sci. 2017; 14: 1181-1188.

4. D'Eliseo D, Velotti F. Omega-3 fatty acids and cancer cell cytotoxicity: implications for multi-targeted cancer therapy. J Clin Med. 2016; 5 pii:E15.

5. Kalmijn S, van Boxtel MP, Ocké M, et al. Dietary intake of fatty acids and fish in relation to cognitive performance at middle age. Neurology. 2004; 62: 275-280.

6. Yang Q, Wang S, Ji Y, et al. Dietary intake of $\omega-3$ PUFAs modifies the absorption, distribution and bioavailability of fatty acids in the mouse gastrointestinal tract. Lipids Health Dis. 2017; 16: 10

7. Borsini A, Alboni S, Horowitz MA, et al. Rescue of IL-1 $\beta$-induced reduction of human neurogenesis by omega- 3 fatty acids and antidepressants. Brain Behav Immun. 2017; 65: 230-238.

8. Mozaffari-Khosravi H, Yassini-Ardakani M, Karamati M, Shariati-Bafghi SE. Eicosapentaenoic acid versus docosahexaenoic acid in mild-to-moderate depression: a randomized, double-blind, placebo-controlled trial. Eur Neuropsychopharmacol. 2013; 23: 636-644.

9. Schunck WH, Konkel A, Fischer R, Weylandt KH. Therapeutic potential of omega-3 fatty acid-derived epoxyeicosanoids in cardiovascular and inflammatory diseases. Pharmacol Ther. 2018; 183: 177-204.

10. Lee CH, Lee SD, Ou HC, Lai SC, Cheng YJ. Eicosapentaenoic acid protects against palmitic acid-induced endothelial dysfunction via activation of the AMPK/eNOS pathway. Int J Mol Sci. 2014; 15: 10334-10349. 
11. Shiau AL, Shen YT, Hsieh JL, Wu CL, Lee CH. Scutellaria barbata inhibits angiogenesis through downregulation of HIF-1 $\alpha$ in lung tumor. Environ Toxicol. 2014; 29: 363-370.

12. Tsao YT, Kuo CY, Kuan YD, Lin HC, Wu LH, Lee CH. The extracts of Astragalus membranaceus inhibit melanogenesis through the ERK signaling pathway. Int J Med Sci. 2017; 14: 1049-1053.

13. Liu WS, Kuan YD, Chiu KH, et al. The extract of Rhodobacter sphaeroides inhibits melanogenesis through the MEK/ERK signaling pathway. Mar Drugs. 2013; 11: 1899-1908.

14. Kuan YD, Lee $\mathrm{CH}$. Salmonella overcomes tumor immune tolerance by inhibition of tumor indoleamine 2, 3-dioxygenase 1 expression. Oncotarget. 2016; 7: 374-385.

15. Munn DH. Indoleamine 2,3-dioxygenase, tumor-induced tolerance and counter-regulation. Curr Opin Immunol. 2006; 18: 220-225.

16. Schley PD, Jijon HB, Robinson LE, Field CJ. Mechanisms of omega-3 fatty acid-induced growth inhibition in MDA-MB-231 human breast cancer cells. Breast Cancer Res Treat. 2005; 92: 187-195.

17. Tsao YT, Huang YF, Kuo CY, et al. Hinokitiol inhibits melanogenesis via AKT/mTOR signaling in B16F10 mouse melanoma cells. Int J Mol Sci. 2016; 17: 248 .

18. Tu DG, Chang WW, Lin ST, Kuo CY, Tsao YT, Lee CH. Salmonella inhibits tumor angiogenesis by downregulation of vascular endothelial growth factor. Oncotarget. 2016; 7: 37513-37523.

19. Bonifazi $\mathrm{P}, \mathrm{D}^{\prime}$ Angelo $\mathrm{C}$, Zagarella $\mathrm{S}$, et al. Intranasal delivered siRNA targeting $\mathrm{PI} 3 \mathrm{~K} / \mathrm{Akt} / \mathrm{mTOR}$ inflammatory pathways protects from aspergillosis. Mucosal Immunol. 2010; 3: 193-205.

20. Lee CH, Lin ST, Liu JJ, Chang WW, Hsieh JL, Wang WK. Salmonella induce autophagy in melanoma by the downregulation of AKT/mTOR pathway. Gene Ther. 2014; 21:309-316.

21. Kolch W, Halasz M, Granovskaya M, Kholodenko BN. The dynamic control of signal transduction networks in cancer cells. Nat Rev Cancer. 2015; 15:515-527.

22. Cao W, Ma Z, Rasenick MM, Yeh S, Yu J. N-3 poly-unsaturated fatty acids shift estrogen signaling to inhibit human breast cancer cell growth. PLoS One. 2012; 7:e52838.

23. Murray M, Hraiki A, Bebawy M, Pazderka C, Rawling T. Anti-tumor activities of lipids and lipid analogues and their development as potential anticancer drugs. Pharmacol Ther. 2015; 150:109-128.

24. Jaudszus A, Gruen M, Watzl B, Ness C, Roth A, Lochner A, Barz D, Gabriel H, Rothe M, Jahreis G. Evaluation of suppressive and pro-resolving effects of EPA and DHA in human primary monocytes and T-helper cells. J Lipid Res. 2013; 54:923-935.

25. Fujikawa M, Yamashita N, Yamazaki K, Sugiyama E, Suzuki H, Hamazaki T. Eicosapentaenoic acid inhibits antigen-presenting cell function of murine splenocytes. Immunology. 1992; 75:330-335.

26. Nakajima K, Yamashita T, Kita T, Takeda M, Sasaki N, Kasahara K, Shinohara M, Rikitake Y, Ishida T, Yokoyama M, Hirata K. Orally administered eicosapentaenoic acid induces rapid regression of atherosclerosis via modulating the phenotype of dendritic cells in LDL receptor-deficient mice. Arterioscler Thromb Vasc Biol. 2011; 31:1963-1972.

27. Polk A, Svane IM, Andersson M, Nielsen D. Checkpoint inhibitors in breast cancer-Current status. Cancer Treat Rev. 2017; 63: 122-134.

28. Hou DY, Muller AJ, Sharma MD, et al. Inhibition of indoleamine 2,3-dioxygenase in dendritic cells by stereoisomers of 1-methyl-tryptophan correlates with antitumor responses. Cancer Res. 2007; 67: 792-801.

29. Muller AJ, DuHadaway JB, Donover PS, Sutanto-Ward E, Prendergast GC. Inhibition of indoleamine 2,3-dioxygenase, an immunoregulatory target of the cancer suppression gene Bin1, potentiates cancer chemotherapy. Nat Med. 2005; 11: 312-319.

30. Fukui M, Kang KS, Okada K, Zhu BT. EPA, an omega-3 fatty acid, induces apoptosis in human pancreatic cancer cells: role of ROS accumulation, caspase- 8 activation, and autophagy induction. J Cell Biochem. 2013; 114: $192-203$ 